\title{
On the Maximum Number of Cliques in a Graph
}

\author{
David R. Wood* \\ Departament de Matemática Aplicada II, Universitat Politècnica de Catalunya, Barcelona, Spain \\ e-mail: david.wood@upc.es
}

\begin{abstract}
A clique is a set of pairwise adjacent vertices in a graph. We determine the maximum number of cliques in a graph for the following graph classes: (1) graphs with $n$ vertices and $m$ edges; (2) graphs with $n$ vertices, $m$ edges, and maximum degree $\Delta ;(3) d$-degenerate graphs with $n$ vertices and $m$ edges; (4) planar graphs with $n$ vertices and $m$ edges; and (5) graphs with $n$ vertices and no $K_{5}$-minor or no $K_{3,3}$-minor. For example, the maximum number of cliques in a planar graph with $n$ vertices is $8(n-2)$.
\end{abstract}

Key words. extremal graph theory, Turán's Theorem, clique, complete subgraph, degeneracy, graph minor, planar graph, $K_{5}$-minor, $K_{3,3}$-minor

\section{Introduction}

The typical question of extremal graph theory asks for the maximum number of edges in a graph in a certain family; see the surveys [2, 38, 39, 40]. For example, a celebrated theorem of Turán [47] states that the maximum number of edges in a graph with $n$ vertices and no $(k+1)$-clique is $\frac{1}{2}\left(1-\frac{1}{k}\right) n^{2}$. Here a clique is a (possibly empty) set of pairwise adjacent vertices in a graph. For $k \geq 0$, a $k$-clique is a clique of cardinality $k$. Since an edge is nothing but a 2 -clique, it is natural to consider the maximum number of $\ell$-cliques in a graph. The following generalisation of Turán's Theorem, first proved by Zykov [52], has been rediscovered and itself generalised by several authors [8, 10, 15, 16, 17, 20, 27, 31, 33, 36].

Theorem 1. ([52]) For all integers $k \geq \ell \geq 0$, the maximum number of $\ell$-cliques in a graph with $n$ vertices and no $(k+1)$-clique is $\left(\begin{array}{c}k \\ \ell\end{array}\right)\left(\frac{n}{k}\right)^{\ell}$.

A simple inductive proof of Theorem 1 is included in Appendix A. In this paper we determine the maximum number of cliques in a graph in each of the following classes:

- graphs with $n$ vertices and $m$ edges (Section 3),

- graphs with $n$ vertices, $m$ edges, and maximum degree $\Delta$ (Section 4 ),

- $d$-degenerate graphs with $n$ vertices and $m$ edges (Section 5),

- planar graphs with $n$ vertices and $m$ edges (Section 6), and

\footnotetext{
* Research supported by a Marie Curie Fellowship of the European Community under contract 023865, and by the projects MCYT-FEDER BFM2003-00368 and Gen. Cat 2001SGR00224.
} 
- graphs with $n$ vertices and no $K_{5}$-minor or no $K_{3,3}$-minor (Section 7 ).

We now review some related work from the literature. Eckhoff [5, 6] determined the maximum number of cliques in a graph with $m$ edges and no $(k+1)$-clique. Lower bounds on the number of cliques in a graph have also been obtained [4, 13, 14, 22, 23, 24, 25]. The number of cliques in a random graph has been studied [3, 29, 37]. Bounds on the number of cliques in a graph have recently been applied in the analysis of an algorithm for finding small separators [32] and in the enumeration of minor-closed families [28].

\section{Preliminaries}

Every graph $G$ that we consider is undirected, finite, and simple. Let $V(G)$ and $E(G)$ be the vertex and edge sets of $G$. Let $\Delta(G)$ be the maximum degree of $G$. We say $G$ is a $(|V(G)|,|E(G)|)$-graph or a $(|V(G)|,|E(G)|, \Delta(G))$-graph.

Let $C(G)$ be the set of cliques in $G$. Let $c(G):=|C(G)|$. Let $C_{k}(G)$ be the set of $k$-cliques in $G$. Let $c_{k}(G):=\left|C_{k}(G)\right|$. Our aim is to prove bounds on $c(G)$ and $c_{k}(G)$.

A clique is not necessarily maximal 1 . In particular, $\emptyset$ is a clique of every graph, $\{v\}$ is a clique for each vertex $v$, and each edge is a clique. Thus every graph $G$ satisfies

$$
c(G) \geq c_{0}(G)+c_{1}(G)+c_{2}(G)=1+|V(G)|+|E(G)| .
$$

A triangle is a 3-clique. Equation (1) implies that

$$
c(G)=1+|V(G)|+|E(G)| \text { if and only if } G \text { is triangle-free. }
$$

Triangle-free graphs have the fewest cliques. Obviously the complete graph $K_{n}$ has the most cliques for a graph on $n$ vertices. In particular, $c\left(K_{n}\right)=2^{n}$ since every set of vertices in $K_{n}$ is a clique.

Say $v$ is a vertex of a graph $G$. Let $G_{v}$ be the subgraph of $G$ induced by the neighbours of $v$. Observe that $X$ is a clique of $G$ containing $v$ if and only if $X=Y \cup\{v\}$ for some clique $Y$ of $G_{v}$. Thus the number of cliques of $G$ that contain $v$ is exactly $c\left(G_{v}\right)$. Every clique of $G$ either contains $v$ or is a clique of $G \backslash v$. Thus $C(G)=C(G \backslash v) \cup\left\{Y \cup\{v\}: Y \in C\left(G_{v}\right)\right\}$ and

$$
c(G)=c(G \backslash v)+c\left(G_{v}\right) \leq c(G \backslash v)+2^{\operatorname{deg}(v)} .
$$

Let $G$ be a graph with induced subgraphs $G_{1}, G_{2}$ and $S$ such that $G=G_{1} \cup G_{2}$ and $G_{1} \cap G_{2}=S$. Then $G$ is obtained by pasting $G_{1}$ and $G_{2}$ on $S$. Observe that $C(G)=$ $C\left(G_{1}\right) \cup C\left(G_{2}\right)$ and $C\left(G_{1}\right) \cap C\left(G_{2}\right)=C(S)$. Thus

$$
c(G)=c\left(G_{1}\right)+c\left(G_{2}\right)-c(S) .
$$

Lemma 1. Let $G$ be an $(n, m)$-graph that is obtained by pasting $G_{1}$ and $G_{2}$ on $S$. Say $G_{i}$ has $n_{i}$ vertices and $m_{i}$ edges. Say $S$ has $n_{S}$ vertices and $m_{S}$ edges. If $c\left(G_{i}\right) \leq x n_{i}+y m_{i}+z$ and $c(S) \geq x n_{s}+y m_{S}+z$, then $c(G) \leq x n+y m+z$.

\footnotetext{
1 Moon and Moser [26] proved that the maximum number of maximal cliques in a graph with $n$ vertices is approximately $3^{n / 3}$; see $[9,11,12,18,19,34,35,42$, 50, 51] for related results.
} 
Proof. By Equation (4),

$$
\begin{aligned}
c(G) & =c\left(G_{1}\right)+c\left(G_{2}\right)-c(S) \\
& \leq\left(x n_{1}+y m_{1}+z\right)+\left(x n_{2}+y m_{2}+z\right)-\left(x n_{s}+y m_{s}+z\right) \\
& =x\left(n_{1}+n_{2}-n_{S}\right)+y\left(m_{1}+m_{2}-m_{S}\right)+z \\
& =x n+y m+z .
\end{aligned}
$$

The following special case of Lemma 1 will be useful.

Corollary 1. Let $G$ be an $(n, m)$-graph that is obtained by pasting $G_{1}$ and $G_{2}$ on a $k$ clique. Say $G_{i}$ has $n_{i}$ vertices and $m_{i}$ edges. Assume that $c\left(G_{i}\right) \leq x n_{i}+y m_{i}+z$ and that $x k+y\left(\begin{array}{c}k \\ 2\end{array}\right)+z \leq 2^{k}$. Then $c(G) \leq x n+y m+z$.

\section{General Graphs}

We now determine the maximum number of cliques in an $(n, m)$-graph.

Theorem 2. Let $n$ and $m$ be non-negative integers such that $m \leq\left(\begin{array}{l}n \\ 2\end{array}\right)$. Let $d$ and $\ell$ be the unique integers such that $m=\left(\begin{array}{l}d \\ 2\end{array}\right)+\ell$ where $d \geq 1$ and $0 \leq \ell \leq d-1$. Then the maximum number of cliques in an $(n, m)$-graph equals $2^{d}+2^{\ell}+n-d-1$.

Proof. First we prove the lower bound. Let $V(G):=\left\{v_{1}, v_{2}, \ldots, v_{n}\right\}$ and $E(G):=\left\{v_{i} v_{j}\right.$ : $1 \leq i<j \leq d\} \cup\left\{v_{i} v_{d+1}: 1 \leq i \leq \ell\right\}$, as illustrated in Figure 1. Then $G$ has $\left(\begin{array}{l}d \\ 2\end{array}\right)+\ell$ edges. Now $\left\{v_{1}, v_{2}, \ldots, v_{d}\right\}$ is a clique, which contains $2^{d}$ cliques (including $\emptyset$ ). The neighbourhood of $v_{d+1}$ is an $\ell$-clique with $2^{\ell}$ cliques. Thus there are $2^{\ell}$ cliques that contain $v_{d+1}$. Finally $v_{d+2}, v_{d+3}, \ldots, v_{n}$ are isolated vertices, which contribute $n-d-1$ cliques to $G$. In total, $G$ has $2^{d}+2^{\ell}+n-d-1$ cliques.

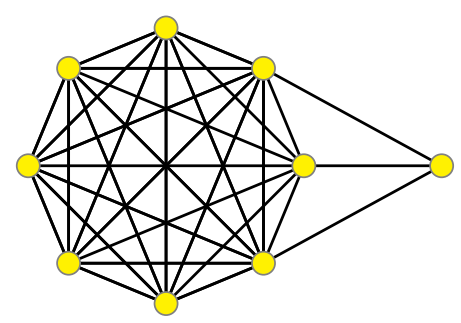

Fig. 1. A (14,31)-graph with 269 cliques $(d=8$ and $\ell=3)$.

Now we prove the upper bound. That is, every $(n, m)$-graph $G$ has at most $2^{d}+2^{\ell}+$ $n-d-1$ cliques. We proceed by induction on $n+m$. For the base case, suppose that $m=0$. Then $d=1, \ell=0$, and $c(G)=n+1=2^{d}+2^{\ell}+n-d-1$. Now assume that $m \geq 1$. Let $v$ be a vertex of minimum degree in $G$. Then $\operatorname{deg}(v) \leq d-1$, as otherwise every vertex has degree at least $d$, implying $m \geq \frac{d n}{2} \geq \frac{d(d+1)}{2}=\left(\begin{array}{c}d+1 \\ 2\end{array}\right)$, which contradicts the definition of $d$. By Equation (3),$c(G) \leq c(G \backslash v)+2^{\operatorname{deg}(v)}$. To apply induction to $G \backslash v$ (which has $n-1$ vertices and $m-\operatorname{deg}(v)$ edges) we distinguish two cases.

First suppose that $\operatorname{deg}(v) \leq \ell$. Thus $m-\operatorname{deg}(v)=\left(\begin{array}{l}d \\ 2\end{array}\right)+\ell-\operatorname{deg}(v)$. By induction, $c(G) \leq 2^{d}+2^{\ell-\operatorname{deg}(v)}+n-1-d-1+2^{\operatorname{deg}(v)}$. Hence the result follows if $2^{d}+2^{\ell-\operatorname{deg}(v)}+$ 
$n-1-d-1+2^{\operatorname{deg}(v)} \leq 2^{d}+2^{\ell}+n-d-1$. That is, $2^{\ell-\operatorname{deg}(v)}-1 \leq\left(2^{\ell-\operatorname{deg}(v)}-1\right) 2^{\operatorname{deg}(v)}$, which is true since $0 \leq \operatorname{deg}(v) \leq \ell$.

Otherwise $\ell+1 \leq \operatorname{deg}(v) \leq d-1$. Thus $m-\operatorname{deg}(v)=\left(\begin{array}{c}d-1 \\ 2\end{array}\right)+d-1+\ell-\operatorname{deg}(v)$. By induction, $c(G) \leq 2^{d-1}+2^{d-1+\ell-\operatorname{deg}(v)}+n-1-d+2^{\operatorname{deg}(v)}$. Hence the result follows if $2^{d-1}+2^{d-1+\ell-\operatorname{deg}(v)}+n-1-d+2^{\operatorname{deg}(v)} \leq 2^{d}+2^{\ell}+n-d-1$. That is, $2^{\ell}\left(2^{\operatorname{deg}(v)-\ell}-1\right) \leq$ $2^{d-1-\operatorname{deg}(v)+\ell}\left(2^{\operatorname{deg}(v)-\ell}-1\right)$. Since $\operatorname{deg}(v) \geq \ell+1$, we need $2^{\ell} \leq 2^{d-1-\operatorname{deg}(v)+\ell}$, which is true since $\operatorname{deg}(v) \leq d-1$.

\section{Bounded Degree Graphs}

We now determine the maximum number of cliques in an $(n, m, \Delta)$-graph. West [49] proved a related result.

Theorem 3. The number of cliques in an $(n, m, \Delta)$-graph $G$ is at most

$$
1+n+\left(\frac{2^{\Delta+1}-\Delta-2}{\left(\begin{array}{c}
\Delta+1 \\
2
\end{array}\right)}\right) m \leq 1+\left(\frac{2^{\Delta+1}-1}{\Delta+1}\right) n .
$$

Proof. $G$ has one 0 -clique and $n$ 1-cliques. For $k \geq 2$, each edge is in at most $\left(\begin{array}{l}\Delta-1 \\ k-2\end{array}\right) k$ cliques, and each $k$-clique contains $\left(\begin{array}{l}k \\ 2\end{array}\right)$ edges. Thus $G$ has at most $m\left(\begin{array}{l}\Delta-1 \\ k-2\end{array}\right) /\left(\begin{array}{l}k \\ 2\end{array}\right) k$-cliques. Thus the number of cliques (not counting 0 - and 1-cliques) is at most

$$
\begin{aligned}
\sum_{k=2}^{\Delta+1} \frac{m\left(\begin{array}{l}
\Delta-1 \\
k-2
\end{array}\right)}{\left(\begin{array}{l}
k \\
2
\end{array}\right)} & =m \sum_{k=2}^{\Delta+1} \frac{2}{k(k-1)} \cdot \frac{(\Delta-1) !}{(k-2) !(\Delta-1-k+2) !} \\
& =\frac{m}{\left(\begin{array}{c}
\Delta+1 \\
2
\end{array}\right)} \sum_{k \geq 2}^{\Delta+1} \frac{2(\Delta-1) !\left(\begin{array}{c}
\Delta+1 \\
2
\end{array}\right)}{k !(\Delta+1-k) !} \\
& =\frac{m}{\left(\begin{array}{c}
\Delta+1 \\
2
\end{array}\right)} \sum_{k=2}^{\Delta+1} \frac{(\Delta+1) !}{k !(\Delta+1-k) !} \\
& =\frac{m}{\left(\begin{array}{c}
\Delta+1 \\
2
\end{array}\right)}\left(\left(\sum_{k=0}^{\Delta+1}\left(\begin{array}{c}
\Delta+1 \\
k
\end{array}\right)\right)-\frac{(\Delta+1) !}{1 !(\Delta+1-1) !}-\frac{(\Delta+1) !}{0 !(\Delta+1-0) !}\right) \\
& =\frac{m}{\left(\begin{array}{c}
\Delta+1 \\
2
\end{array}\right)}\left(2^{\Delta+1}-\Delta-2\right) .
\end{aligned}
$$

The result follows since $m \leq \frac{\Delta n}{2}$.

The bound in Theorem 3 is tight for many values of $m$.

Proposition 1. For all $n$ and $m$ such that $m \leq \frac{\Delta n}{2}$ and $m \equiv 0\left(\bmod \left(\begin{array}{c}\Delta+1 \\ 2\end{array}\right)\right)$, there is an $(n, m, \Delta)$-graph $G$ with

$$
c(G)=1+n+\left(\frac{2^{\Delta+1}-\Delta-2}{\left(\begin{array}{c}
\Delta+1 \\
2
\end{array}\right)}\right) m .
$$

Proof. Let $p:=m /\left(\begin{array}{c}\Delta+1 \\ 2\end{array}\right)$. Let $G$ consist of $p$ copies of $K_{\Delta+1}$, plus $n-p(\Delta+1)$ isolated vertices. Then $G$ is an $(n, m, \Delta)$-graph. Each copy of $K_{\Delta+1}$ contributes $2^{\Delta+1}-\Delta-2$ cliques with at least two vertices. Thus $G$ has $1+n+\left(2^{\Delta+1}-\Delta-2\right) p$ cliques. 


\section{Degenerate Graphs}

A graph $G$ is $d$-degenerate if every subgraph of $G$ has a vertex with degree at most $d$. The following simple result is well known; see [7, 32] for example.

Proposition 2. Every d-degenerate graph $G$ with $n \geq d$ vertices has at most $2^{d}(n-d+1)$ cliques.

Proof. We proceed by induction on $n$. If $n=d$ then $c(G) \leq 2^{d}=2^{d}(n-d+1)$. Now assume that $n \geq d+1$. Let $v$ be a vertex of $G$ with $\operatorname{deg}(v) \leq d$. By Equation (3), $c(G) \leq c(G \backslash v)+2^{\operatorname{deg}(v)}$. Now $G \backslash v$ is $d$-degenerate since it is a subgraph of $G$. Moreover, $G \backslash v$ has at least $d$ vertices. By induction, $c(G \backslash v) \leq 2^{d}(n-1-d+1)$. Thus $c(G) \leq$ $2^{d}(n-1-d+1)+2^{d}=2^{d}(n-d+1)$.

The bound in Proposition 2 is tight.

Proposition 3. For all $n \geq d$, there is a d-degenerate graph $G_{n}$ with $n$ vertices and exactly $2^{d}(n-d+1)$ cliques (and with a d-clique).

Proof. Let $G_{d}$ be the complete graph $K_{d}$. Then $G_{d}$ has the desired properties. For $n \geq$ $d+1$, let $G_{n}$ be the graph obtained by adding one new vertex $v$ adjacent to every vertex in some $d$-clique in $G_{n-1}$. Then $G_{n}$ is $d$-degenerate and contains a $d$-clique. $\left(G_{n}\right.$ is a chordal graph called a $d$-tree; see [1].) By Equation (3]), $c\left(G_{n}\right)=c\left(G_{n-1}\right)+2^{\operatorname{deg}(v)}=$ $2^{d}(n-1-d+1)+2^{d}=2^{d}(n-d+1)$.

Proposition 2 can be made sensitive to the number of edges as follows.

Theorem 4. For all $d \geq 1$, every d-degenerate graph $G$ with $n$ vertices and $m \geq\left(\begin{array}{l}d \\ 2\end{array}\right)$ edges has at most

$$
n+\frac{\left(2^{d}-1\right) m}{d}-\frac{(d-3) 2^{d}+d+1}{2}
$$

cliques.

Proof. We proceed by induction on $n+m$. For the base case, suppose that $m=\left(\begin{array}{l}d \\ 2\end{array}\right)+\ell$ where $d \geq 1$ and $0 \leq \ell \leq d-1$. Thus $c(G) \leq 2^{d}+2^{\ell}+n-d-1$ by Theorem 2, and the result follows if

$$
2^{d}+2^{\ell}+n-d-1 \leq n+\frac{\left(2^{d}-1\right) m}{d}-\frac{(d-3) 2^{d}+d+1}{2} .
$$

That is, $d\left(2^{\ell}-1\right) \leq \ell\left(2^{d}-1\right)$, which we prove in Lemma 2 below.

Now assume that $m \geq\left(\begin{array}{c}d+1 \\ 2\end{array}\right)$. Now $G$ has a vertex $v$ with $\operatorname{deg}(v) \leq d$. By Equation (3), $c(G) \leq c(G \backslash v)+2^{\operatorname{deg}(v)}$. The graph $G \backslash v$ has $m-\operatorname{deg}(v) \geq\left(\begin{array}{l}d \\ 2\end{array}\right)$ edges, and is $d$-degenerate since it is a subgraph of $G$. By induction,

$$
c(G \backslash v) \leq n-1+\frac{\left(2^{d}-1\right)(m-\operatorname{deg}(v))}{d}-\frac{(d-3) 2^{d}+d+1}{2} .
$$

Thus the result follows if

$$
-1+\frac{\left(2^{d}-1\right)(m-\operatorname{deg}(v))}{d}+2^{\operatorname{deg}(v)} \leq \frac{\left(2^{d}-1\right) m}{d} .
$$

That is, $d\left(2^{\operatorname{deg}(v)}-1\right) \leq\left(2^{d}-1\right) \operatorname{deg}(v)$, which holds by Lemma 2 below. 
Lemma 2. $d\left(2^{\ell}-1\right) \leq \ell\left(2^{d}-1\right)$ for all integers $d \geq \ell \geq 0$.

Proof. The case $\ell=0$ is trivial. Now assume that $\ell \geq 1$. We proceed by induction on $d$. The base case $d=\ell$ is trivial. Assume that $d \geq \ell+1 \geq 2$ and by induction,

$$
(d-1)\left(2^{\ell}-1\right) \leq \ell\left(2^{d-1}-1\right) .
$$

Since $d \geq 2$,

$$
\frac{d}{d-1} \leq 2<2+\frac{1}{2^{d-1}-1}=\frac{2^{d}-1}{2^{d-1}-1} .
$$

Equations (5) and (6) imply that

$$
(d-1)\left(2^{\ell}-1\right) \cdot \frac{d}{d-1}<\ell\left(2^{d-1}-1\right) \cdot \frac{2^{d}-1}{2^{d-1}-1} .
$$

That is, $d\left(2^{\ell}-1\right)<\ell\left(2^{d}-1\right)$, as desired.

Note that a $d$-degenerate $n$-vertex graph has at most $d n-\left(\begin{array}{c}d+1 \\ 2\end{array}\right)$ edges, and Theorem 4 with $m=d n-\left(\begin{array}{c}d+1 \\ 2\end{array}\right)$ is equivalent to Proposition 2.

The bound in Theorem 4 is tight for many values of $m$.

Proposition 4. Let $d \geq 1$. For all $n$ and $m$ such that $\left(\begin{array}{l}d \\ 2\end{array}\right) \leq m \leq d n-\left(\begin{array}{c}d+1 \\ 2\end{array}\right)$ and

$$
m \bmod d= \begin{cases}0 & \text { if } d \text { is odd } \\ \frac{d}{2} & \text { if } d \text { is even },\end{cases}
$$

there is a d-degenerate $(n, m)$-graph $G$ with

$$
c(G)=n+\frac{\left(2^{d}-1\right) m}{d}-\frac{(d-3) 2^{d}+d+1}{2} .
$$

Proof. Let $n^{\prime}:=\frac{m}{d}+\frac{1}{2}(d+1)$. Then $n^{\prime}$ is an integer and $d \leq n^{\prime} \leq n$. Let $G$ consist of a $d$-degenerate $n^{\prime}$-vertex graph with $2^{d}\left(n^{\prime}-d+1\right)$ cliques (from Proposition 3), plus $n-n^{\prime}$ isolated vertices. Then $G$ has $m$ edges and $c(G)=2^{d}\left(n^{\prime}-d+1\right)+n-n^{\prime}=$ $n+\left(2^{d}-1\right) \frac{m}{d}-\frac{1}{2}\left((d-3) 2^{d}+d+1\right)$.

A graph is 1-degenerate if and only if it is a forest. Thus Theorem 4 with $d=1$ implies that every forest has at most $n+m-1$ cliques, which also follows from Equation (2). In particular, $c(T)=2 n$ for every $n$-vertex tree $T$.

Theorem 4 with $d=2$ implies that every 2 -degenerate graph has at most $n+\frac{1}{2}(3 m+1)$ cliques. Outerplanar graphs are 2-degenerate. The construction in Propositions 3 and 4 can produce outerplanar graphs. (Add each new vertex adjacent to two consecutive vertices on the outerface.) Thus this bound is tight for outerplanar graphs.

\section{Planar Graphs}

Papadimitriou and Yannakakis [30] and Storch [44] proved that every $n$-vertex planar graph has $\mathcal{O}(n)$ cliques; see [7] for a more general result. The proof is based on the corollary of Euler's Formula that planar graphs are 5-degenerate. By Theorem 4, if $G$ is a planar $(n, m)$-graph with $m \geq 10$, then $c(G)<n+\frac{31}{5} m<\frac{98}{5} n$. We now prove that the bound for 3-degenerate graphs in Theorem 4 also holds for planar graphs. 
Theorem 5. Every planar $(n, m)$-graph $G$ with $m \geq 3$ has at most $n+\frac{7}{3} m-2$ cliques.

Proof. We proceed by induction on $n+m$. The result is easily verified if $m=3$.

Suppose that $G$ has a separating triangle $T$. Thus $G$ is obtained by pasting two induced subgraphs $G_{1}$ and $G_{2}$ on $T$. Say $G_{i}$ has $n_{i}$ vertices and $m_{i}$ edges. Then $m_{i} \geq 3$ since $T \subset G_{i}$. By induction, $c\left(G_{i}\right) \leq n_{i}+\frac{7}{3} m_{i}-2$. By Corollary 1 with $k=3, x=1, y=\frac{7}{3}$ and $z=-2$, we have $c(G) \leq n+\frac{7}{3} m-2\left(\right.$ since $\left.1 \cdot 3+\frac{7}{3}\left(\begin{array}{l}3 \\ 2\end{array}\right)-2=2^{3}\right)$. Now assume that $G$ has no separating triangle.

Let $v$ be a vertex of $G$. We have $c(G)=c(G \backslash v)+c\left(G_{v}\right)$ by Equation (3). The graph $G \backslash v$ has $m-\operatorname{deg}(v)$ edges. Suppose that $m-\operatorname{deg}(v) \leq 2$. (Then we cannot apply induction to $G \backslash v$.) Then $G$ has no 4 -clique and at most two triangles. If $G$ has at most one triangle, then $c(G) \leq 1+n+m+1 \leq n+\frac{7}{3} m-2$ since $m \geq 3$. Otherwise $G$ has two triangles, and $c(G) \leq 1+n+m+2<n+\frac{7}{3} m-2$ since $m \geq 5$.

Now assume that $m-\operatorname{deg}(v) \geq 3$. By (3) , applying induction to $G \backslash v$,

$$
c(G)=c(G \backslash v)+c\left(G_{v}\right) \leq(n-1)+\frac{7}{3}(m-\operatorname{deg}(v))-2+c\left(G_{v}\right) .
$$

Fix a plane embedding of $G$. If $u w$ is an edge of $G_{v}$, then the edges $v u$ and $v w$ are consecutive in the circular ordering of edges incident to $v$ defined by the embedding (as otherwise $G$ would contain a separating triangle). Thus $\Delta\left(G_{v}\right) \leq 2$ and $c\left(G_{v}\right) \leq$ $1+\frac{7}{3} \operatorname{deg}(v)$ by Theorem 3. Hence

$$
c(G) \leq(n-1)+\left(\frac{7}{3}(m-\operatorname{deg}(v))-2\right)+\left(1+\frac{7}{3} \operatorname{deg}(v)\right)=n+\frac{7}{3} m-2 .
$$

If $n \geq 3$ in Theorem 5 then $m \leq 3(n-2)$ by Euler's Formula. Thus we have the following corollary.

Corollary 2. Every planar graph with $n \geq 3$ vertices has at most $8(n-2)$ cliques.

We now prove bounds on the number of 3- and 4-cliques in a planar graph.

Proposition 5. For every planar graph $G$ with $n \geq 3$ vertices, $c_{3}(G) \leq 3 n-8$ and $c_{4}(G) \leq n-3$.

Proof. We proceed by induction on $n$. The result is trivial if $n \leq 4$. Now assume that $n \geq 5$. First suppose that $G$ has no separating triangle. Then $c_{4}(G)=0$, and every triangle of $G$ is a face. By Euler's Formula, $c_{3}(G) \leq 2 n-4<3 n-8$ faces. Now suppose that $G$ has a separating triangle $T$. Thus $G$ is obtained by pasting two induced subgraphs $G_{1}$ and $G_{2}$ on $T$. Say $G_{i}$ has $n_{i}$ vertices. Then $n_{i} \geq 3$ since $T \subset G_{i}$. By induction, $c_{3}\left(G_{i}\right) \leq 3 n_{i}-8$ and $c_{4}\left(G_{i}\right) \leq n_{i}-3$. Every clique of $G$ is a clique of $G_{1}$ or $G_{2}$. Thus $c_{4}(G)=c_{4}\left(G_{1}\right)+c_{4}\left(G_{2}\right) \leq n_{1}-3+n_{2}-3=n-3$. Moreover, $T$ is a triangle in both $G_{1}$ and $G_{2}$. Thus $c_{3}(G) \leq\left(3 n_{1}-8\right)+\left(3 n_{2}-8\right)-1=3\left(n_{1}+n_{2}\right)-17=3(n+3)-17=3 n-8$.

Note that Proposition 5 and Euler's Formula (which implies $c_{2}(G) \leq 3 n-6$ ) reprove Corollary 2, since $1+n+3(n-2)+(3 n-8)+(n-3)=8(n-2)$.

We now show that all our bounds for planar graphs are tight.

Proposition 6. For all $n \geq 3$ there is a maximal planar $n$-vertex graph $G_{n}$ with $c_{2}\left(G_{n}\right)=$ $3(n-2), c_{3}\left(G_{n}\right)=3 n-8, c_{4}\left(G_{n}\right)=n-3$, and $c\left(G_{n}\right)=8(n-2)$. 
Proof. Let $G_{3}:=K_{3}$. Then $c_{2}\left(G_{3}\right)=3, c_{3}\left(G_{3}\right)=1, c_{4}\left(G_{3}\right)=0$, and $c\left(G_{3}\right)=8$. Say $G_{n-1}$ is a maximal planar $(n-1)$-vertex graph with $c_{2}\left(G_{n-1}\right)=3(n-3), c_{3}\left(G_{n-1}\right)=3 n-11$, $c_{4}\left(G_{n-1}\right)=n-4$, and $c\left(G_{n}\right)=8(n-3)$. Let $G_{n}$ be the maximal planar $n$-vertex graph obtained by adding one new vertex $v$ adjacent to each vertex of some face of $G_{n-1}$, as illustrated in Figure 2, Then $c_{2}\left(G_{n}\right)=c_{2}\left(G_{n-1}\right)+3=3(n-2), c_{3}\left(G_{n}\right)=c_{3}\left(G_{n-1}\right)+3=$ $3 n-8, c_{4}\left(G_{n}\right)=c_{4}\left(G_{n-1}\right)+1=n-3$, and $c\left(G_{n}\right)=c\left(G_{n-1}\right)+c\left(G_{n}(v)\right)=8(n-3)+8=$ $8(n-2)$. (Note that $G_{n}$ is also an example of a 3-degenerate graph with the maximum number of cliques; see Proposition [3.)

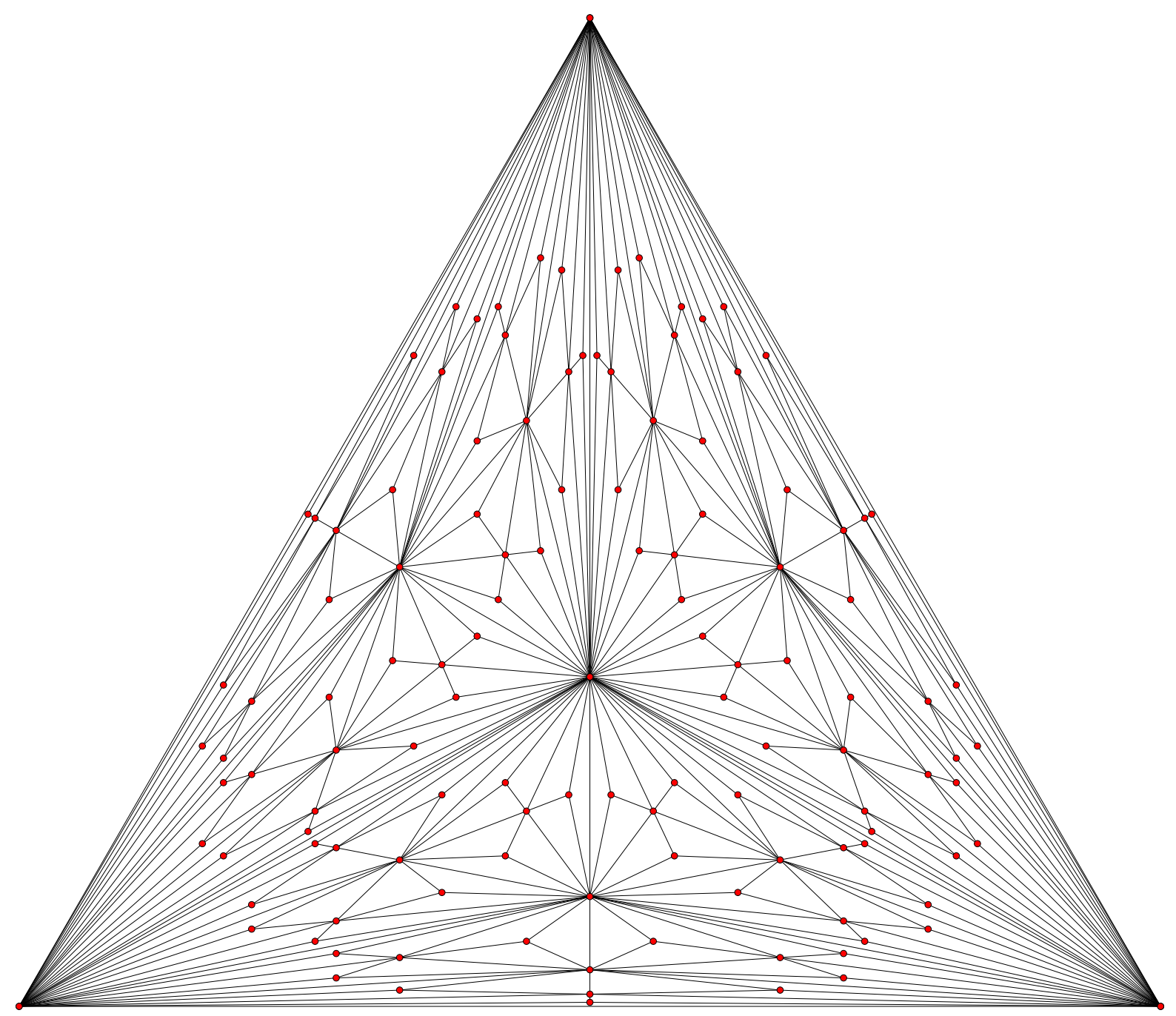

Fig. 2. A planar graph with 124 vertices, 366 edges, 364 triangles, 121 4-cliques, and 976 cliques. It is obtained by repeatedly adding one degree- 3 vertex inside each internal face (starting from $\left.K_{3}\right)$.

Proposition 7. For all $n \geq 3$ and $m \in\{3,6, \ldots, 3 n-6\}$, there is a planar $(n, m)$-graph $G$ with $c(G)=n+\frac{7}{3} m-2$. 
Proof. Let $n^{\prime}:=\frac{m}{3}+2$. Let $G$ consist of a maximal planar graph on $n^{\prime}$ vertices with $8\left(n^{\prime}-2\right)$ cliques (from Proposition [6), plus $n-n^{\prime}$ isolated vertices. Then $G$ has $n$ vertices and $m$ edges, and $c(G)=8\left(n^{\prime}-2\right)+n-n^{\prime}=n+7 n^{\prime}-16=n+7\left(\frac{m}{3}+2\right)-16=n+\frac{7}{3} m-2$.

\section{Graphs with no $K_{5}$-Minor}

A graph $H$ is a minor of a graph $G$ if $H$ can be obtained from a subgraph of $G$ by contracting edges. The graphs with no $K_{3}$-minor are the forests, which have at most $2 n$ cliques, and this bound is tight. The graphs with no $K_{4}$-minor (called series-parallel) are 2-degenerate, and thus have at most $4(n-1)$ cliques, and this bound is tight. The Kuratowski-Wagner Theorem characterises planar graphs as those with no $K_{5}$-minor and no $K_{3,3}$-minor. We now extend Corollary 2 for graphs with no $K_{5}$-minor (but possibly a $K_{3,3}$-minor).

Theorem 6. Every graph $G$ with $n \geq 3$ vertices and no $K_{5}$-minor has at most $8(n-2)$ cliques.

Proof. Let $V_{8}$ be the graph obtained from the 8-cycle by adding an edge between each pair of antipodal vertices; see Figure 3, Let $G$ be a minimum counterexample to the theorem. We can assume that $G$ is edge-maximal with no $K_{5}$-minor. Wagner [48] proved that (a) $G$ is a maximal planar graph, (b) $G=V_{8}$, or (c) $G$ is obtained by pasting two smaller graphs (that are thus not counterexamples), each with no $K_{5}$-minor, on an edge or a triangle $T$. In case (a) the result is Corollary 2, In case (b), since $V_{8}$ is triangle-free, $c\left(V_{8}\right)=1+\left|V\left(V_{8}\right)\right|+\left|E\left(V_{8}\right)\right|=21<8\left(\left|V\left(V_{8}\right)\right|-2\right)$ by Equation (2). In case (c), if $T$ is an edge, we have $c(G) \leq 8(n-2)$ by Corollary 1 with $k=2, x=8, y=0$ and $z=-16$ (since $\left.8 \cdot 2+0-16<2^{2}\right)$. In case $(\mathrm{c})$, if $T$ is a triangle, we have $c(G) \leq 8(n-2)$ by Corollary 1 with $k=3, x=8, y=0$ and $z=-16$ (since $8 \cdot 3+0-16=2^{3}$ ).

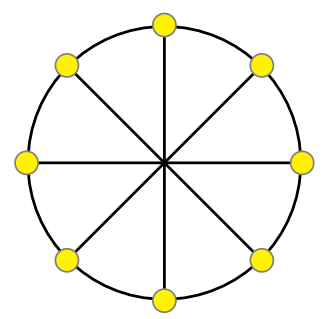

Fig. 3. The graph $V_{8}$.

A similar result is obtained for graphs with no $K_{3,3}$-minor.

Theorem 7. Every graph $G$ with $n \geq 3$ vertices and no $K_{3,3}$-minor has at most $\frac{4}{3}(7 n-11)$ cliques. Conversely, for all $n \equiv 2(\bmod 3)$ with $n \geq 5$ there is an $n$-vertex graph with no $K_{3,3}$-minor and $c(G)=\frac{4}{3}(7 n-11)$.

Proof. Let $G$ be a minimum counterexample. We can assume that $G$ is edge-maximal with no $K_{3,3}$-minor. Wagner [48] proved that (a) $G$ is a maximal planar graph, (b) $G=K_{5}$, or (c) $G$ is obtained by pasting two smaller graphs (that are thus not counterexamples), each with no $K_{3,3}$-minor, on an edge. In case (a) the result follows from Corollary 2 since 
$8 n-16<\frac{4}{3}(7 n-11)$. In case $(\mathrm{b}), c\left(K_{5}\right)=32=\frac{4}{3}(7 \cdot 5-11)$. In case (c), we have $c(G) \leq \frac{4}{3}(7 n-11)$ by Corollary 1 with $k=2, x=\frac{28}{3}, y=0$ and $z=-\frac{44}{3}$ (since $\frac{28}{3} \cdot 2+0-\frac{44}{3}=2^{2}$ ). By the same analysis, the graph obtained from $K_{5}$ by repeatedly pasting copies of $K_{5}$ on an edge has no $K_{3,3}$-minor and $\frac{4}{3}(7 n-11)$ cliques.

We finish with an open problem: What is the maximum number of cliques in an $n$ vertex graph $G$ with no $K_{t}$-minor? Kostochka [21] and Thomason [45] independently proved that $G$ is $\mathcal{O}(t \sqrt{\log t})$-degenerate 2 . Thus Proposition 2 implies that $G$ has at most $2^{\mathcal{O}(t \sqrt{\log t})} n$ cliques; similar bounds can be found in [28, 32]. It is unknown whether this bound can be improved to $c^{t} n$ for some constant $c$ (possibly for sufficiently large $n$ ).

We have proved that $c(G) \leq 2^{t-2}(n-t+3)$ whenever $t \leq 5$. Moreover, the graph $G$ in Proposition 3 (with $t=d+2$ ) has no $K_{t}$-minor and $c(G)=2^{t-2}(n-t+3$ ). However, for large values of $t$ this upper bound does not hold for the complete $k$-partite graph $K_{2,2, \ldots, 2}$. By Theorem 8 in Appendix B, the maximum order of a clique minor in $K_{2,2, \ldots, 2}$ is $\left\lfloor\frac{3}{2} k\right\rfloor$. But by Proposition [10, $c\left(K_{2,2, \ldots, 2}\right)=3^{k}>2^{\lfloor 3 k / 2\rfloor-1}\left(2 k-\left\lfloor\frac{3}{2} k\right\rfloor+2\right)$ for all $k \geq 42$.

Acknowledgements. Thanks to a referee for pointing out reference $\left[\frac{12}{12}\right]$.

\section{References}

1. Hans L. Bodlaender. A partial $k$-arboretum of graphs with bounded treewidth. Theoret. Comput. Sci., 209(1-2):1-45, 1998.

2. BÉla Bollobás. Extremal graph theory. In Handbook of combinatorics, Vol. 1, 2, pp. 1231-1292. Elsevier, 1995.

3. BÉla Bollobás And Paul Erdős. Cliques in random graphs. Math. Proc. Cambridge Philos. Soc., 80(3):419-427, 1976.

4. BÉla Bollobás, Paul Erdős, and Endre Szemerédi. On complete subgraphs of $r$-chromatic graphs. Discrete Math., 13(2):97-107, 1975.

5. Jürgen ECKhoff. The maximum number of triangles in a $K_{4}$-free graph. Discrete Math., 194(1-3):95-106, 1999.

6. Jürgen Eckhoff. A new Turán-type theorem for cliques in graphs. Discrete Math., 282(1-3):113-122, 2004.

7. DAvid Eppstein. Connectivity, graph minors, and subgraph multiplicity. J. Graph Theory, 17(3):409-416, 1993.

8. PAul ERDös. On the number of complete subgraphs contained in certain graphs. Magyar Tud. Akad. Mat. Kutató Int. Közl., 7:459-464, 1962.

9. PAul ERdős. On cliques in graphs. Israel J. Math., 4:233-234, 1966.

10. PAul ERDős. On the number of complete subgraphs and circuits contained in graphs. Časopis Pěst. Mat., 94:290-296, 1969.

11. Cong Fan And Jiuqiang Liu. On cliques of graphs. In Graph theory, Combinatorics, Algorithms, and Applications, pp. 140-150. SIAM, 1991.

12. Martin Farber, Mihály Hujter, and Zsolt Tuza. An upper bound on the number of cliques in a graph. Networks, 23(3):207-210, 1993.

13. DAvid C. Fisher. Lower bounds on the number of triangles in a graph. J. Graph Theory, 13(4):505-512, 1989.

${ }^{2}$ Moreover, this bound is best possible; Thomason [46] even determined the asymptotic constant. 
14. David C. Fisher And Jason M. Nonis. Bounds on the "growth factor" of a graph. In Proc. 21st Southeastern Conf. on Combinatorics, Graph Theory, and Computing, vol. 77 of Congr. Numer., pp. 113-119. Utilitas Math., 1990.

15. David C. Fisher And Jennifer Ryan. Bounds on the number of complete subgraphs. Discrete Math., 103(3):313-320, 1992.

16. Nikola ̌ G. HadžIivanov. Maximum of a multilinear form of integer variables, and its application in the theory of extremal graphs. C. R. Acad. Bulgare Sci., 30(10):1373-1376, 1977.

17. Nikola ̌ G. Hadžitvanov and Nedyalko D. Nenov. p-sequences of graphs and some extremal properties of Turán graphs. C. R. Acad. Bulgare Sci., 30(4):475-478, 1977.

18. Frank Harary And Abraham Lempel. On clique-extremal $(p, q)$-graphs. Networks, 4:371-378, 1974.

19. Min-Jen Jou And Gerard J. Chang. The number of maximum independent sets in graphs. Taiwanese J. Math., 4(4):685-695, 2000.

20. Nikola Ĭ Khadzhitvanov And Nedyalko Nenov. Sharp estimates of the highest number of cliques of a graph. Annuaire Univ. Sofia Fac. Math. Méc., 70:23-26, $1975 / 76$.

21. Alexandr V. Kostochka. The minimum Hadwiger number for graphs with a given mean degree of vertices. Metody Diskret. Analiz., 38:37-58, 1982.

22. David G. Larman. On the number of complete subgraphs and circuits in a graph. Proc. Roy. Soc. Ser. A, 308:327-342, 1969.

23. LÁszló Lovász ANd Miklós Simonovits. On the number of complete subgraphs of a graph. In Proc. of 5th British Combinatorial Conference, vol. XV of Congr. Numer., pp. 431-441. Utilitas Math., 1976.

24. LÁszló Lovász And Miklós Simonovits. On the number of complete subgraphs of a graph. II. In Studies in pure mathematics, pp. 459-495. Birkhäuser, 1983.

25. John W. Moon. On the number of complete subgraphs of a graph. Canad. Math. Bull., 8:831-834, 1965.

26. John W. Moon And Leo Moser. On cliques in graphs. Israel J. Math., 3:23-28, 1965.

27. Nedyalko Nenov and Nikola ̌ Khadzhitvanov. A new proof of a theorem about the maximum number of $p$-cliques of graphs. Annuaire Univ. Sofia Fac. Math. Méc., 70:93-98, 1975/76.

28. Serguei Norine, Paul Seymour, Robin Thomas, and Paul Wollan. Proper minor-closed families are small. J. Combin. Theory Ser. B, 96(5):754-757, 2006.

29. Daniel Olejár and Eduard Toman. On the order and the number of cliques in a random graph. Math. Slovaca, 47(5):499-510, 1997.

30. Christos H. Papadimitriou and Mihalis Yannakakis. The clique problem for planar graphs. Inform. Process. Lett., 13(4-5):131-133, 1981.

31. Louis Petingi And Jose Rodriguez. A new proof of the Fisher-Ryan bounds for the number of cliques of a graph. In Proc. 31st Southeastern International Conf. on Combinatorics, Graph Theory and Computing, vol. 146 of Congr. Numer., pp. 143-146. 2000.

32. Bruce Reed and David R. Wood. Fast separation in a graph with an excluded minor. In Proc. European Conf. on Combinatorics, Graph Theory and Applications (EuroComb '05), vol. AE of Discrete Math. Theor. Comput. Sci. Proceedings, pp. 
45-50. 2005.

33. Steven Roman. The maximum number of $q$-cliques in a graph with no $p$-clique. Discrete Math., 14(4):365-371, 1976.

34. Bruce E. Sagan And Vincent R. Vatter. Maximal and maximum independent sets in graphs with at most $r$ cycles. J. Graph Theory, 53(4):283-314, 2006.

35. Iwao Sato. Clique graphs of packed graphs. Discrete Math., 62(1):107-109, 1986.

36. Norbert Sauer. A generalization of a theorem of Turán. J. Combinatorial Theory Ser. B, 10:109-112, 1971.

37. Klaus Schürger. Limit theorems for complete subgraphs of random graphs. Period. Math. Hungar., 10(1):47-53, 1979.

38. Miklós Simonovits. Extremal graph theory. In Selected topics in graph theory, 2, pp. 161-200. Academic Press, 1983.

39. Miklós Simonovits. Paul Erdős' influence on extremal graph theory. In The mathematics of Paul Erdös, II, vol. 14 of Algorithms Combin., pp. 148-192. Springer, 1997.

40. Miklós Simonovits And Vera T. Sós. Ramsey-Turán theory. Discrete Math., 229(1-3):293-340, 2001.

41. David SitTon. Maximum matchings in complete multipartite graphs. Furman University Electronic J. Undergraduate Math., 2:6-16, 1996.

42. Joel H. Spencer. On cliques in graphs. Israel J. Math., 9:419-421, 1971.

43. Michael Stiebitz. On Hadwiger's number - a problem of the Nordhaus-Gaddum type. Discrete Math., 101(1-3):307-317, 1992.

44. Tobias Storch. How randomized search heuristics find maximum cliques in planar graphs. In Proc. of 8th Annual Conf. on Genetic and Evolutionary Computation (GECCO '06), pp. 567-574. ACM Press, 2006.

45. Andrew Thomason. An extremal function for contractions of graphs. Math. Proc. Cambridge Philos. Soc., 95(2):261-265, 1984.

46. Andrew Thomason. The extremal function for complete minors. J. Combin. Theory Ser. B, 81(2):318-338, 2001.

47. Paul Turán. On an extremal problem in graph theory. Mat. Fiz. Lapok, 48:436-452, 1941.

48. Klaus Wagner. Über eine Eigenschaft der ebene Komplexe. Math. Ann., 114:570 $590,1937$.

49. Douglas B. West. The number of complete subgraphs in graphs with nonmajorizable degree sequences. In Progress in graph theory, pp. 509-521. Academic Press, 1984.

50. Herbert S. Wilf. The number of maximal independent sets in a tree. SIAM J. Algebraic Discrete Methods, 7(1):125-130, 1986.

51. Goh Chee Ying, Koh Khee Meng, Bruce E. Sagan, and Vincent R. VatTER. Maximal independent sets in graphs with at most $r$ cycles. J. Graph Theory, $53(4): 270-282,2006$.

52. Alexander A. Zykov. On some properties of linear complexes. Mat. Sbornik N.S., 24(66):163-188, 1949.

\section{A. Graphs with Bounded Cliques}

In this appendix we give a simple inductive proof of Theorem 1 , 
Proposition 8. For all integers $k \geq \ell \geq 0$, every graph $G$ with $n \geq \ell$ vertices and no $(k+1)$-clique has at most $\left(\begin{array}{c}k \\ \ell\end{array}\right)\left(\frac{n}{k}\right)^{\ell} \ell$-cliques.

Proof. We proceed by induction on $n$. For the base case, suppose that $n \leq k$. Trivially $c_{\ell}(G) \leq\left(\begin{array}{c}n \\ \ell\end{array}\right)$, which is at most $\left(\begin{array}{c}k \\ \ell\end{array}\right)\left(\frac{n}{k}\right)^{\ell}$ by Lemma 3 below. Now assume that the result holds for graphs with less than $n$ vertices, and $n>k$. Let $G$ be a graph with $n$ vertices, no $(k+1)$-clique, and with $c_{\ell}(G)$ maximum. We can add edges to $G$ until it contains a $k$-clique $X$. Every $\ell$-clique of $G$ is the union of some $i$-clique of $G \backslash X$ and some $(\ell-i)$ clique of $G[X]$, for some $0 \leq i \leq \ell$. Moreover, the vertices in each $i$-clique of $G \backslash X$ have at most $k-i$ common neighbours in $X$ (since $X$ is a clique and $G$ has no $(k+1)$-clique). Thus from each $i$-clique of $G \backslash X$, we obtain at most $\left(\begin{array}{c}k-i \\ \ell-i\end{array}\right) \ell$-cliques of $G$. By induction, $c_{i}(G \backslash X) \leq\left(\begin{array}{c}k \\ i\end{array}\right)\left(\frac{n-k}{k}\right)^{i}$. Thus

$$
c_{\ell}(G) \leq \sum_{i=0}^{\ell}\left(\begin{array}{l}
k \\
i
\end{array}\right)\left(\frac{n-k}{k}\right)^{i}\left(\begin{array}{l}
k-i \\
\ell-i
\end{array}\right)=\left(\begin{array}{l}
k \\
\ell
\end{array}\right) \sum_{i=0}^{\ell}\left(\begin{array}{l}
\ell \\
i
\end{array}\right)\left(\frac{n}{k}-1\right)^{i}=\left(\begin{array}{l}
k \\
\ell
\end{array}\right)\left(\frac{n}{k}\right)^{\ell}
$$

by the binomial theorem ${ }^{3}$.

Lemma 3. $\left(\begin{array}{c}n \\ \ell\end{array}\right) k^{\ell} \leq\left(\begin{array}{c}k \\ \ell\end{array}\right) n^{\ell}$ for all integers $k \geq n \geq \ell \geq 0$.

Proof. We proceed by induction on $\ell$. The claim is trivial with $\ell=0$. Now assume that $\ell \geq 1$. Thus $k-n \leq \ell(k-n)$, implying $k n+k-n \leq k n+\ell(k-n)$. That is, $k(n-\ell+1) \leq n(k-\ell+1)$. By induction,

$$
\left(\begin{array}{c}
n \\
\ell-1
\end{array}\right) k^{\ell-1} \cdot k(n-\ell+1) \leq\left(\begin{array}{c}
k \\
\ell-1
\end{array}\right) n^{\ell-1} \cdot n(k-\ell+1) .
$$

That is,

$$
\frac{n ! k^{\ell}(n-\ell+1)}{(n-\ell+1) !(\ell-1) !} \leq \frac{k ! n^{\ell}(k-\ell+1)}{(k-\ell+1) !(\ell-1) !} .
$$

Hence

$$
\frac{n ! k^{\ell}}{(n-\ell) ! \ell !} \leq \frac{k ! n^{\ell}}{(k-\ell) ! \ell !}
$$

as desired.

Proposition 9. Every graph $G$ with $n$ vertices and no $(k+1)$-clique has at most $\left(\frac{n}{k}+1\right)^{k}$ cliques.

Proof. By Proposition 8 and the binomial theorem,

$$
c(G) \leq \sum_{\ell=0}^{k}\left(\begin{array}{l}
k \\
\ell
\end{array}\right)\left(\frac{n}{k}\right)^{\ell}=\left(\frac{n}{k}+1\right)^{k}
$$

We now prove that Propositions 8 and 9 are tight.

3 Twice we use that $x^{t}=\sum_{j=0}^{t}\left(\begin{array}{l}t \\ j\end{array}\right)(x-1)^{j}$ for all real $x$. 
Proposition 10. For every complete $k$-partite graph $G=K_{n_{1}, n_{2}, \ldots, n_{k}}$,

$$
c(G)=\prod_{i=1}^{k}\left(n_{i}+1\right) .
$$

In particular, if every $n_{i}=\frac{n}{k}$ then $c(G)=\left(\frac{n}{k}+1\right)^{k}$ and $c_{\ell}(G)=\left(\begin{array}{c}k \\ \ell\end{array}\right)\left(\frac{n}{k}\right)^{\ell}$ whenever $0 \leq \ell \leq k$.

Proof. Every clique consists of at most one vertex from each of the $k$ colour classes. There are $n_{i}+1$ ways to choose at most one vertex from the $i$-th colour class. Thus $c(G)=\prod_{i}\left(n_{i}+1\right)$. (This result can also be proved using Equation (3).) Now assume that every $n_{i}=\frac{n}{k}$. Every $\ell$-clique consists of exactly one vertex from each of $\ell$ colour classes.

There are $\left(\begin{array}{l}k \\ \ell\end{array}\right)$ ways to choose $\ell$ colour classes and $\frac{n}{k}$ ways to choose exactly one vertex from each colour class. Each combination gives a distinct $\ell$-clique. The result follows.

It is interesting to note that the extremal examples in Proposition 1 for graphs of bounded degree (disjoint copies of cliques) are the complements of the extremal examples in Proposition 10 for graphs with bounded cliques (complete multipartite graphs).

\section{B. Clique Minors in a Complete Multipartite Graph}

The Hadwiger number of a graph $G$, denoted by $\eta(G)$, is the maximum order of a clique minor in $G$. Stiebitz [43] proved that $\eta(G) \leq \frac{1}{2}(n+k)$ for every $n$-vertex graph $G$ with no $(k+1)$-clique. We now prove that this bound is tight for every complete $k$-partite graph if the largest colour class is not too large.

Theorem 8. Let $G$ be a complete $k$-partite graph on $n$ vertices with $n^{\prime}$ vertices in the largest colour class. Then $\eta(G)=\min \left\{\frac{1}{2}(n+k), n-n^{\prime}+1\right\}$.

The proof of Theorem 8 is based on the following lemma.

Lemma 4. Let $G$ be the complete $k$-partite graph $K_{n_{1}, n_{2}, \ldots, n_{k}}$ with each $n_{i} \geq 1$. Then $\eta(G)$ equals $k$ plus the size of the largest matching in $G^{\prime}:=K_{n_{1}-1, n_{2}-1, \ldots, n_{k}-1}$.

Proof. Consider $G^{\prime}$ to be a subgraph of $G$, so that $S:=V(G) \backslash V\left(G^{\prime}\right)$ is a $k$-clique of $G$. Let $M$ be a matching of $G^{\prime}$. If $v$ is a vertex and $e$ is an edge of $G^{\prime}$, then $v$ is adjacent to at least one endpoint of $e$. Thus every vertex in $S$ is adjacent to at least one endpoint of every edge in $M$, and for all edges $e$ and $f$ in $M$, at least one endpoint of $e$ is adjacent to at least one endpoint of $f$. Thus by contracting each edge of $M$ within $G$, we obtain a $K_{k+|M|}$-minor in $G$.

Now suppose that $K_{t}$ is a minor of $G$ with $t$ maximum. Then $G$ has disjoint vertex sets $X_{1}, X_{2}, \ldots, X_{t}$, such that each $X_{i}$ induces a connected subgraph of $G$, and for all $i \neq j$, some vertex in $X_{i}$ is adjacent to some vertex in $X_{j}$.

Suppose that some $X_{i}$ contains two vertices $v$ and $w$ in the same colour class of $G$. Since $v$ and $w$ have the same neighbourhood, we can delete $w$ from $X_{i}$ and still have a $K_{t}$-minor. Now assume that the vertices in each set $X_{i}$ are from distinct colour classes.

Suppose that some $X_{i}$ contains at least three vertices $u, v, w$. Since the neighbourhood of $u$ is contained in the union of the neighbourhoods of $v$ and $w$, we can delete $u$ from $X_{i}$ and still have a $K_{t}$-minor. Now assume that each set $X_{i}$ has cardinality 1 or 2 . 
Suppose that for some colour class $\ell$, no set $X_{i}$ contains a vertex coloured $\ell$. Then $X_{1}, \ldots, X_{t}$ along with a set consisting of one vertex coloured $\ell$ forms a $K_{t+1}$-minor, which is a contradiction. Now assume that for every colour class $\ell$, there is some set $X_{i}$ that contains a vertex coloured $\ell$.

Suppose that for some colour class $\ell$, every set $X_{i}$ that contains some vertex coloured $\ell$ has cardinality 2. Let $X_{i}=\{v, w\}$ be such a set, where $v$ is coloured $\ell$. Thus $v$ is adjacent to some vertex in every set $X_{j}$. Thus we can delete $w$ from $X_{i}$ and still have a $K_{t}$-minor. Now assume that for each colour class $\ell$, some set $X_{i}$ consists of one vertex coloured $\ell$. No two singleton sets $X_{i}$ and $X_{j}$ contain vertices of the same colour. Thus there are $k$ singleton sets $X_{i}$, one for each colour class. The remaining sets $X_{i}$ thus form a matching in $G^{\prime}$.

Proof of Theorem 8. Sitton [41] proved that the size of the largest matching in a complete multipartite graph on $n$ vertices with $n^{\prime}$ vertices in the largest colour class is $\min \left\{\left\lfloor\frac{n}{2}\right\rfloor, n-n^{\prime}\right\}$. Applying this result to the graph $G^{\prime}$ in Lemma 4.

$$
\eta(G)=k+\min \left\{\frac{1}{2}(n-k),(n-k)-\left(n^{\prime}-1\right)\right\}=\min \left\{\frac{1}{2}(n+k), n-n^{\prime}+1\right\} .
$$

Received: June 23, 2006 